\title{
Searches for light charged Higgs bosons decaying to a hadronic $\tau$ and a neutrino in the one lepton mode with ATLAS
}

Thies Ehrich* on behalf of the ATLAS Collaboration

Max-Planck-Institut für Physik, München

E-mail: thies.ehrich@cern.ch

Light charged Higgs bosons could be produced in $t \bar{t}$ decay and the final state consists of a lepton, a $\tau$-jet, $2 b$-jets and several neutrinos. The latter makes the reconstruction of the complete event impossible. However, in the Minimal Supersymmetic extension of the Standard Model (MSSM) the branching ratio of the decay $H^{ \pm} \rightarrow \tau v$ is close to one for a wide parameter space and the signal appears as an excess of $\tau$ leptons in the final state over the main background (Standard Model $t \bar{t}$ events). Here, an ATLAS simulation study for this channel is introduced, problematic aspects are outlined, and the expected $H^{ \pm}$sensitivity within the $\mathrm{m}_{\mathrm{h}}$-max scenario of the MSSM is shown.

Prospects for Charged Higgs Discovery at Colliders

September 16-19 2008

Uppsala, Sweden

\footnotetext{
* Speaker.
} 


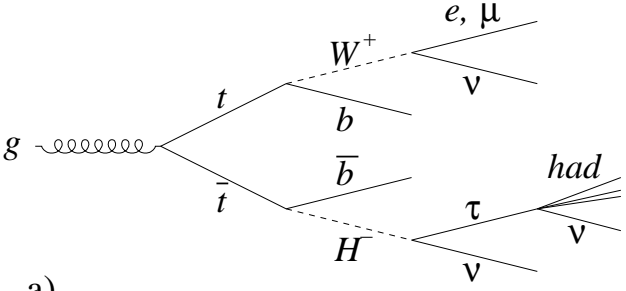

a)

Figure 1: Signal (a) and main $t \bar{t}$ background (b)

\section{Signal and Backgrounds}

This analysis investigates the discovery potential of the light charged Higgs boson below the top quark mass threshold. It is part of Higgs studies done in ATLAS [1]. Light charged Higgs bosons are produced in on-shell top quark decays $t \rightarrow H^{ \pm} b$, followed by the decay $H^{ \pm} \rightarrow \tau \nu$. In the $\mathrm{m}_{\mathrm{h}^{-}}$ max scenario of the MSSM the latter process is the dominant one with a branching ratio of $\sim 100 \%$ for $\tan \beta>3$ [2]. In this study the $\tau$-lepton decays into a jet (see Fig.1). Since at LHC top quarks will be mainly produced pairwise, we have the possibility to use leptonic $W$ decays ( $W \rightarrow e v$ or $\mu v)$ of the other top quark to trigger the events.

The natural background of this process is Standard Model $t \bar{t}$ production with two $W$ bosons, one decaying into electron or muon final states and the other one into a $\tau$-jet. Because of the presence of three neutrinos distributed over both top quark decay chains, it is impossible to reconstruct the full event, also the top quarks or the $W$ boson can't be reconstructed. Hence, no mass related variable with a distinct shape can be extracted to discriminate the signal from background. The observation of a signal is only possible as an overall excess of $\tau$ events over the irreducible SM $t \bar{t}$ background. Another important but reducible background is the SM $t \bar{t}$ production with misidentified $\tau$-jets. This misidentification happens mostly due to the presence of light jets or misreconstructed electrons in the event. Less important backgrounds are single top and $W+$ Jets productions. These backgrounds may also contribute due to misidentified particles, but show less missing transverse energy than charged Higgs boson events. Another possible source of background, that we expect to be negligible with respect to the ones mentioned above, is represented by QCD processes. Studies are still ongoing, as large Monte Carlo samples are needed to get an accurate estimate of this contamination.

While all backgrounds expected to be important are covered, two sources of signal aren't considered in this study: Charged Higgs boson production is also possible in single top events, but these are suppressed due to the selection cuts. In addition the $t \bar{t}$ mode with a $W$ boson decaying into $\tau$-leptons $(W \rightarrow \tau \nu)$ isn't taken into account in the signal event generation. This mode contributes to the signal both through leptonic and hadronic $\tau$ decays if the charged Higgs boson decays appropriately. In this context the signal yields are underestimated and the results of this study are correspondingly conservative. 

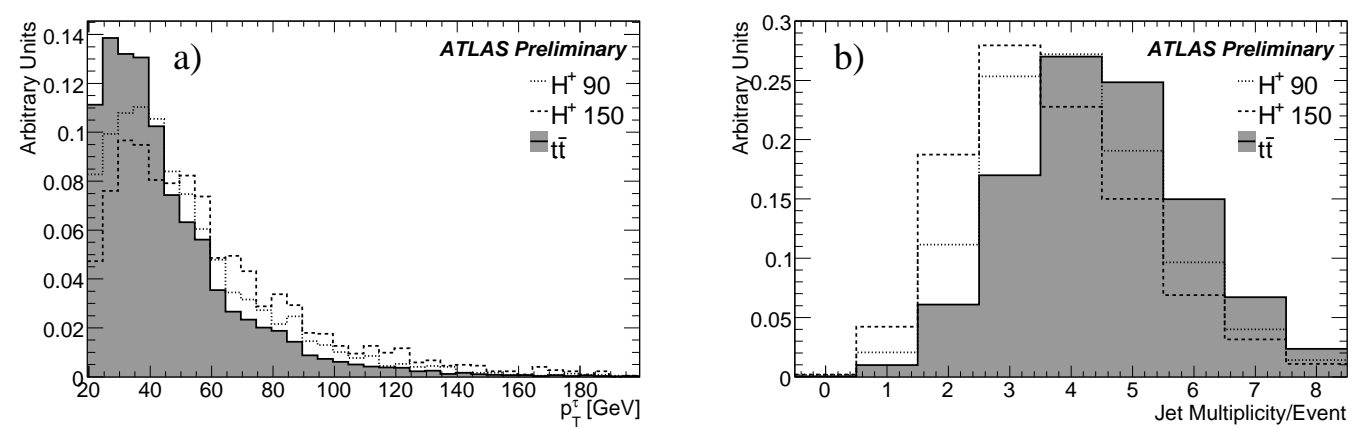

Figure 2: (a) $p_{T}^{\tau}$ and (b) jet multiplicity for two signal samples $\left(\mathrm{m}_{H^{ \pm}}=90,150 \mathrm{GeV}\right)$ and the $t \bar{t}$ background.

\section{Event Selection}

The events are triggered with single lepton triggers, and additional requirements are made on missing transverse energy:

- isolated electron, $p_{T}>22 \mathrm{GeV},|\eta|<2.5+\mathrm{E}_{\mathrm{T}}^{\text {miss }}>30 \mathrm{GeV}$;

- isolated muon, $p_{T}>20 \mathrm{GeV},|\eta|<2.5+\mathrm{E}_{\mathrm{T}}^{\text {miss }}>30 \mathrm{GeV}$.

In addition also $\tau$ trigger signatures will be used:

- isolated $\tau$-jet, $p_{T}>30 \mathrm{GeV},|\eta|<2.5+\mathrm{E}_{\mathrm{T}}^{\text {miss }}>40 \mathrm{GeV}+3$ jets $\left(p_{T}>20 \mathrm{GeV},|\eta|<5\right)$;

- isolated $\tau$-jet, $p_{T}>30 \mathrm{GeV},|\eta|<2.5+\mathrm{E}_{\mathrm{T}}^{\text {miss }}>50 \mathrm{GeV}$.

The lepton has to be reconstructed by the offline algorithms with a transverse momentum $p_{T}>25 \mathrm{GeV}$ for electrons or $p_{T}>20 \mathrm{GeV}$ for muons within a pseudorapidity range $|\eta|<2.5$. Furthermore the lepton has to be isolated to reject events with leptonic $B$ meson decays. For the muons the energy in the calorimeters in a cone with a radius $\mathrm{R}<0.3$ is required to be smaller than $9 \mathrm{GeV}$. The energy in a cone around the electron $(\mathrm{R}<0.2)$ is calculated from the tracks and has to be smaller than $6 \mathrm{GeV}$. In both cases the energy of the particle itself was substracted from the energy in the cone.

A cut is placed on the number of jets being at least three with a transverse momentum $p_{T}>20 \mathrm{GeV}$ and $|\eta|<5$. Because of the fact that $\mathrm{m}_{H^{ \pm}}<\mathrm{m}_{\text {top }}$ one higher energetic jet with a $p_{T}>40 \mathrm{GeV}$ has to be tagged as a $\tau$-jet (see Fig. 2(a)) by a dedicated $\tau$ identification algorithm [3] within $|\eta|<2.5$. Because many events with misidentified $\tau$-jets come from electrons, all $\tau$-jets overlapping an electron (not necessarily isolated) are removed. A further rejection of unidentified electrons is obtained by the cut $E_{T} / p_{T}$ of the leading track being bigger than 0.1 .

To reduce the $W+$ jets background $b$-tagging techniques are used to intentify jets stemming from $b$ quarks [1]. In principle two $b$-jets are present in the signal and $t \bar{t}$ background, but since $\mathrm{m}_{H^{ \pm}}>\mathrm{m}_{W}$ the charged Higgs boson accompanying $b$-jet tends to be softer than $b$-jets in Standard Model $t \bar{t}$ decays. Because the reconstruction efficiency for jets decreases for low $p_{T}$ a smaller fraction of $b$-jets is reconstructed in signal events compared to Standard Model $t \bar{t}$ events. This effect gets more pronounced for heavier $H^{ \pm}$bosons, as shown in Fig.2(b). In addition also the efficiency for identifying 


\begin{tabular}{ll||r|r|r|r|r|r|r|r|r} 
Channel & & All ev. & Trigger & $\geq 1 e, \mu$ & $\geq 3$ jets & $\geq 1 \tau$ & $\geq 1 b$ & $\tau p_{T}$ & $\sum \mathrm{q}$ & $\mathrm{E}_{\mathrm{T}}^{\text {miss }}$ \\
\hline$H^{ \pm}(110 \mathrm{GeV})$ & {$[\mathrm{fb}]$} & 8570 & 4510 & 3534 & 2986 & 772 & 650 & 439 & 431 & 30 \\
& {$[/]$} & & 0.53 & 0.78 & 0.84 & 0.26 & 0.84 & 0.67 & 0.98 & 0.07 \\
$t \bar{t}(\geq 1 \mathrm{lep})$ & {$[\mathrm{fb}]$} & 452000 & 169612 & 137928 & 122547 & 4760 & 4006 & 1915 & 1730 & 78 \\
& {$[/]$} & & 0.37 & 0.81 & 0.89 & 0.04 & 0.84 & 0.48 & 0.90 & 0.04 \\
\multirow{4}{*}{ single top } & {$[\mathrm{fb}]$} & \multirow{2}{*}{112500} & 30180 & 25065 & 18081 & 271 & 168 & 47 & 38 & - \\
& {$[/]$} & & 0.27 & 0.83 & 0.72 & 0.02 & 0.61 & 0.28 & 0.81 & - \\
$W \rightarrow l v+$ jets & {$[\mathrm{fb}]$} & \multirow{2}{*}{769547} & 216556 & 166598 & 101473 & 1549 & 180 & 92 & 58 & - \\
& {$[/]$} & & 0.28 & 0.77 & 0.61 & 0.02 & 0.12 & 0.51 & 0.63 & -
\end{tabular}

Table 1: Cutflow for the signal and backgrounds. The numbers are normalized to cross sections, for the signal a charged Higgs boson mass of $\mathrm{m}_{H^{ \pm}}=110 \mathrm{GeV}$ is considered and $\tan \beta$ is set to 20 . The remaining SUSY parameters are set according to the $\mathrm{m}_{\mathrm{h}}$-max benchmark scenario. Below the cross section the relative cut efficiencies (in italics) are given.
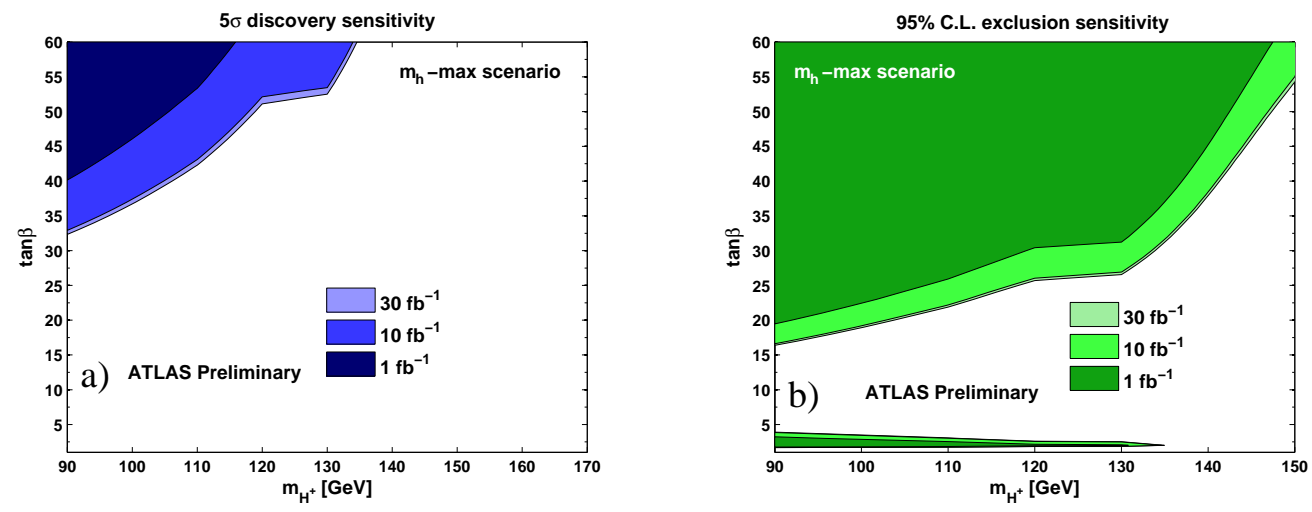

Figure 3: (a) Discovery contour and (b) exclusion limits for the $m_{h}$-max SUSY benchmark scenario, statistical and systematic uncertainties are included.

the $b$-jet correctly drops at low $p_{T}$. Thus only one $b$-jet is required to be $b$-tagged, which takes these effects into account.

Furthermore the electric charge of the reconstructed lepton and $\tau$-jet are required to be opposite, because $t \bar{t}$ quark pairs are neutral. Finally the neutrinos from a charged Higgs boson and its $\tau$ jet are expected to be higher energetic than those from a $W$, in the same way that the $\tau$ itself was. So at least a missing transverse energy $\mathrm{E}_{\mathrm{T}}^{\mathrm{miss}}$ of $175 \mathrm{GeV}$ is demanded. The latter $\mathrm{E}_{\mathrm{T}}^{\text {miss }}$ and the $p_{T}^{\tau}$ cut values where optimized taking into account systematic uncertainties. In Table 1 the cutflow is given for one signal and the considered backgrounds. It is evident that the identification of the $\tau$-jet is the most effective tool to reject reducible backgrounds. Also the cuts on $p_{T}^{\tau}$ and $\mathrm{E}_{\mathrm{T}}^{\text {miss }}$ enhance the signal over the background as expected.

In the $\mathrm{m}_{\mathrm{h}}$-max SUSY benchmark scenario [1] with a charged Higgs boson mass $\mathrm{m}_{H^{ \pm}}=110 \mathrm{GeV}$ and $\tan \beta=20$ we expect, for $1 \mathrm{fb}^{-1}, 30$ signal and $78 t \bar{t}$ events. It has to be noted that the latter number is given for the Standard Model case, in the MSSM scenario one would expect only 72 events due to the reduced branching ratio of the $t \rightarrow W b$ decay. 


\section{Systematic Uncertainties}

The effect of various expected systematic uncertainties has been evaluated for this analysis. It has been found that the dominating experimental uncertainty is given by the jet energy scale due to the measurement of the missing transverse energy $\mathrm{E}_{\mathrm{T}}^{\mathrm{miss}}$. This is estimated to be $40 \%$, while other uncertainties like the $\tau$-jet energy scale or $\tau$-jet energy resolution are only of the order of $10 \%$. The theoretical uncertainty on the $t \bar{t}$ cross-section is $12 \%$, whereas the uncertainties on the branching ratios of the decays $t \rightarrow H^{ \pm} b$ and $H^{ \pm} \rightarrow \tau \nu$ are smaller than $10 \%$ and 5\% respectively. To get a better knowledge of the level of the $t \bar{t}$ background a method was developed to measure it directly from the data itself. It was shown that this method works with an accuracy of $10 \%$ [5], hence this value is used for the background uncertainty when calculating the discovery and exclusion limits.

\section{Results}

The limits for discovery and exclusion of $H^{ \pm}$are shown in Fig. 3(a) and 3(b) in the $\mathrm{m}_{H^{ \pm}-\tan \beta}$ plane for the $\mathrm{m}_{\mathrm{h}}$-max SUSY benchmark scenario. The statistical and systematic uncertainties are included in the calculation of these contours. It can be seen that in this channel charged Higgs bosons can be observed for $\tan \beta$ values greater than 35 for an integrated luminosity of $10 \mathrm{fb}^{-1}$. The exclusion is possible for a wide $\tan \beta$ region, but remains uncovered around 7.5. This parameter space is experimentally hard to reach due to weak $\mathrm{tb} H^{ \pm}$couplings. For higher integrated luminosities the results shown are conservative due to the limited size of the Monte Carlo samples. Here the limits are significantly degraded by the statistical uncertainty.

\section{Summary and Conclusion}

The ATLAS limits for the discovery and exclusion of the light charged Higgs boson in the $\mathrm{m}_{\mathrm{h}^{-}}$ max scenario of the MSSM have been evaluated for a final state containing a lepton and a $\tau$-jet. Because of the high branching ratio of the decay $H^{ \pm} \rightarrow \tau \nu$ being close to one for $\tan \beta>3$ the charged Higgs boson would become manifest in an excess of events over the irreducible Standard Model $t \bar{t}$ background. For the lowest mass of $90 \mathrm{GeV} \tan \beta$ values bigger than 30 can already be excluded with a small integrated luminosity of $1 \mathrm{fb}^{-1}$, provided that the higher level reconstruction objects used in this analysis are already well optimized at that time. The most important tool is the identification algorithm for $\tau$-jets, also $b$-tagging and the measurement of the missing transverse energy $\mathrm{E}_{\mathrm{T}}^{\text {miss }}$ have to be well understood. A prerequisite towards a $H^{ \pm}$discovery in this channel is the measurement of the Standard Model $t \bar{t}$ background from data. While the estimation of the irreducible mode with genuine $\tau$-jets is already approached, modes with fake $\tau$-jets are not studied yet.

I would like to thank Susanne Mohrdieck-Möck for guidance and the charged Higgs group, especially Martin Flechl, Elias Coniavitis, Andre Sopczak, Ofer Vitells and Chris Potter for discussions and good cooperation. Also, I would like to thank the organizers of the conference for hospitality and the nice atmosphere in Uppsala. 


\section{References}

[1] ATLAS Collaboration, Expected Performance of the ATLAS Experiment, Detector, Trigger and Physics, CERN-OPEN-2008-020, Geneva, 2008, to appear

[2] A. Sopczak, Cross-sections and branching ratios for charged Higgs searches, this volume

[3] M. Heldmann and D. Cavalli, An Improved $\tau$-Identification for the ATLAS Experiment, ATL-PHYS-PUB-2006-008, 2006

[4] G. Piacquadio, B-Tagging in ATLAS: expected performance and its calibration in data, this volume

[5] T. Vickey, Data-driven Methods for the Estimation of $t \bar{t}$ Backgrounds to Charged Higgs Searches, this volume 